\title{
The Load Forecasting Model Design of Power System Based on Intelligent Algorithm
}

\author{
Han $\operatorname{Han}^{1, a}$ \\ ${ }^{1}$ Electric power system and automation specialty of Hohai University, Nanjing Jiangsu 210000,China \\ a253198419@qq.com
}

Keywords: Intelligent algorithm; power load; forecasting model; RBF neural network

\begin{abstract}
The paper aims at improving control of power plants on the system. Inaccurate predicting outcomes or oversize errors will impact fuel reasonable allocation of a power-generation department. In order to promote safe and economic operation of power grid, the paper analyzes power load forecasting, constructs a main framework of start grid load forecasting based on the cloud computing, uses fuzzy control theory to adjust and modify RBF neural network model, to improve the rate of convergence and to reduce training time, as well as establishes a load forecasting model of power system combining RBF neural network model and fuzzy control. Forecasting outcomes show that application of the model combining RBF neural network and fuzzy control generates minimum errors. The forecasting effects will be better, indicating the method has practice significance on load forecasting of power system.
\end{abstract}

\section{Introduction}

Load forecasting is the foundation on realization of optimizing power system and has a prominent influence on safety, reliability and economy of power system ${ }^{[1,2,3]}$. With the development of grid intelligent degree, first-tier cities are faced with millions of power data during the peak period of power utilization. One-year data storage scale will be increased to TB or even PB from the current GB. Meanwhile, data dimension of power load is also transmitted from dozens to hundreds ${ }^{[4]}$.The bottleneck that computing resources of one machine are insufficient will be encountered when traditional load forecasting is carried forward under such high-dimensional data. Cloud computing technology has stronger adaptation for processing high-dimensional data and real-time solution and provides real-time, reliable and cheap computing resources for the algorithm consuming lots of resources.

The paper utilizes BP neural network model, RBF neural network model and the model combining RBF neural network with fuzzy control to predict the actual load in an area and to analyze and contrast errors for results. The precision of these forecasting outcomes from these methods can satisfy actual requirements of electric power department, indicating their effectiveness and practicability. Forecasting outcomes show that application of the model combining RBF neural network and fuzzy control generates minimum errors. The forecasting effects will be better, indicating the method has practice significance on load forecasting of power system.

\section{Load Forecasting of Smart Grid}

In terms of load forecasting, there are numerous systems for analysis and predication, and these systems have totally different characteristics. With the rapid development of information technologies application in electric power industry, the insufficiency of traditional load forecasting under the requirements of the "informatization" in smart grid should be overcome. Moreover, it is necessary to establish a load forecasting information service platform. Load analysis and forecasting are based on the data integrity and reliability ${ }^{[5,6]}$. Load forecasting should cater to main characteristics of smart grid, while basic facilities of smart grid and all kinds of information service platforms provide a powerful safeguard on load forecasting. Load forecasting is an important constituent part and basic content of smart grid, which is the data mining system environment and 
technical support of load forecasting. Thus, smart grid and load forecasting are inseparable and organic wholes. To sum up, studying load forecasting of smart grid is significant.

The development of smart grid brings a brand-new challenge. Under the environment of smart grid, the research objects of power load forecasting not only include traditional objects, such as areas, busbar and big consumers, but also involve in distributed generated output, new energy and power forecasting of intelligent terminal. The load forecasting integration of smart grid not only reflects in studying intelligent forecasting algorithm, but also fully utilizes various comprehensive information platforms. The load forecasting of smart grid not only changes in connotations, but also expands outwards, such as wind speed forecasting in wind energy predication. Compared with traditional load forecasting, load forecasting of smart grid presents the following characteristics: it impacts factor diversification for load forecasting of smart grid; forecasting objects of smart grid are diversified; and load forecasting technology is more intelligent and has adaptivity. Load forecasting of smart grid has higher requirements for precision and density and needs to establish intelligent information base.

From the perspective of predicating process, the main framework for load forecasting of smart grid includes abnormal data processing of initial intelligence and sample screening, the forecasting model selection of middle intelligence and forecasting result modification of final intelligence. The load forecasting of smart grid establishes the total electrical load about relevant relations of influences; constructs the uncontrolled load about relevant relations of influences; and analyzes changing disciplines of network supply/controlled loads in line with the relation of "total electrical load=network supply/controlled loads+ uncontrolled loads.” Load forecasting cloud of smart grid is the private cloud facing to load analysis, prediction, management and outcome coordination. At present, business cloud with the priority to Google and Amazon is widely applied to e-commerce and communication, etc., fields. Moreover, it can't be used for load forecasting cloud computing platform of power system directly. The load forecasting cloud framework of smart grid is shown in Figure 1.

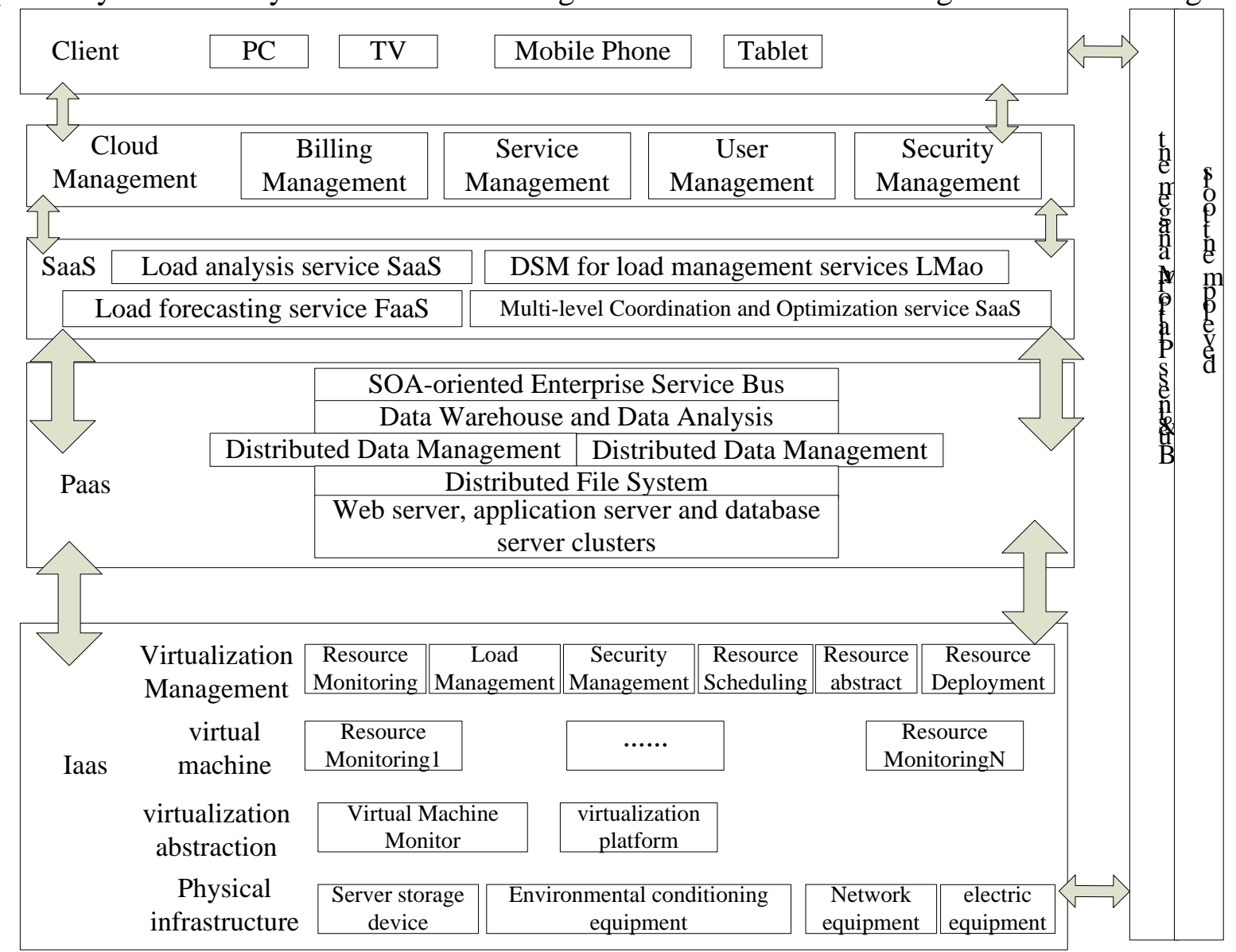

Fig. 1 Load Forecasting Cloud of Smart Grid

The entire platform is mainly divided into "cloud", “end”, support of corresponding management business and development tools. These development tools include Hadoop cloud computing system 
suite(Linux operating system, Hadoop+ HDFS cloud computing system and Java6 development procedures, and so on), virtual platforms(Virtual Box, VMware, Xen and KVM etc., ) MapReduce procedure development tools Eclipse+ IBM MapReduce Tools Eclipse Plugins etc. The "end” refers to the "client". Users of different majors in dispatcher control centers at all or different levels(regulatory major, mode major, communication major, automation specialty, comprehensive major and relaying major) send requires to the load forecasting cloud and acquire services by using the terminal equipment("four screens"-computers, televisions, mobile phones and IPAD through the internet(by using currently brand-new internet technology to make remote cloud services through the internet possible and establish a bridge between users and cloud).

\section{Load Forecasting of Power System Combining RBF Neural Network and Fuzzy Control}

\section{Design of Fuzzy Controller}

Fuzzy control combines the fuzzy set theory and automatic control theory, uses a computer to establish fuzzy control rules, as well as utilizes these rules to replace human control experience, so as to realize non-linear control. For fuzzy control system, language control rules should be expressed as the numerical value. "IF-THEN" sentence should be used to analyze and judge. Finally, output can be obtained. The core part of fuzzy control system is the fuzzy controller, which regards output of controlled objects as the controlled quantity. Through feedback, the controlled quantity is compared with the given input quantity, obtains error amount and considers it as the input. Steps of fuzzy control algorithm are shown as follows: Sampling information is applied to acquire output value and calculate systematic input, changes input into fuzzy quantity; reasons controlled quantity through fuzzy control in line with the input and controlled rules, and finally calculates accurate controlled quantity. Fuzzy controller is an important constituent part of fuzzy control. The structure is shown as the Figure 2.

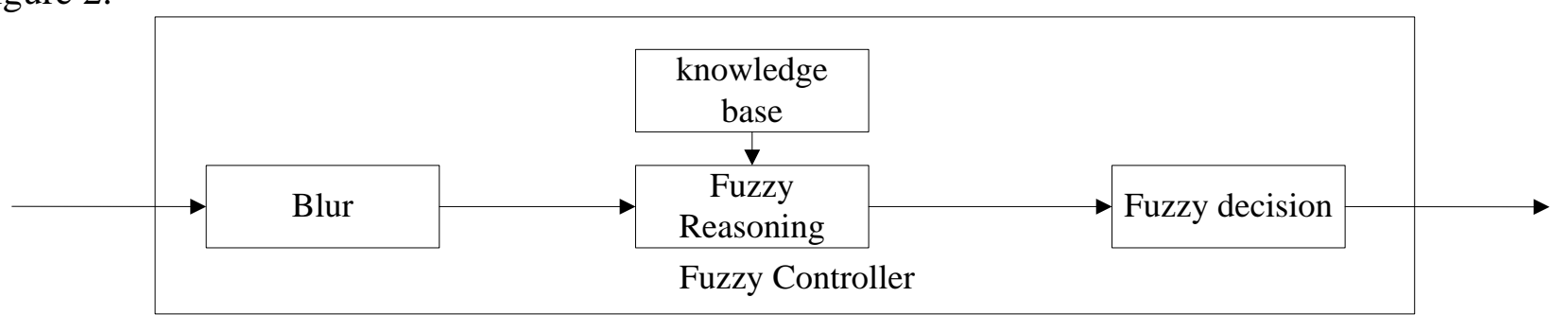

Fig. 2 Composition Diagram of Fuzzy Controller

For selecting input and output linguistic variables of the fuzzy controller, it is necessary to consider the specific situations. Fuzzy controller is a systematic model established on teh basis of stimulating human experience. Moreover, it is the feedback control and regards output of controlled objects as the controlled quantity.

For the entire system, deviation e between output and expected value and deviation change rate ec or accumulated deviation Jedt as the input, and controlled quantity is considered as the output. The controller that uses such a controlled method is the double-input and single-output controller. For different situations, no matter deviation e and deviation change rate ec are considered as input, or no matter deviation e and accumulated deviation fedt are considered as input, it should depend on some situations. In practice, most of situations use deviation e and devation change rate ec as input, and controlled quantity $U$ is considered as output. The method basically can satisfy requirements of the control system.

In practice, it is proved that mrmbrtdhip function shape has a smaller influence on the control system. Thus, the triangular membership function is generally utilized. Fig. 3 provides a typical memebership funtion distribution of the linguistic variable. 


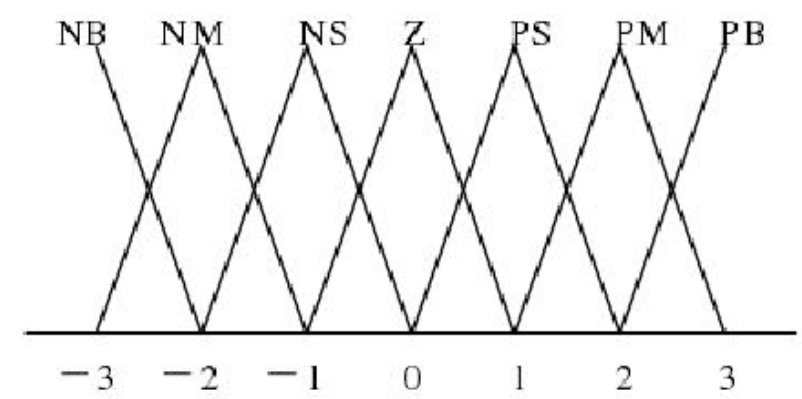

Fig. 3 Membership Function Distribution of the Linguistic Variable

After selecting a linguistic variable, membership function is confirmed. The selection will impact the effects of control system. The general principles of membership function selection are shown as follows: It is necessary to ensure that fuzzy set only has a peak value with the number of 1 ; The stack range of membership function ranges from $25 \%$ to $75 \%$, making membership function abide by language rules; it is necessary to ensure that membership function distribution of all fuzzy linguistic variables will covers the entire domain of discourse. Quantification factor is a numerical value that converts input variable from the basic domain of discourse to the fuzzy set domain. When conducting fuzzy process, input variable should multiply the quantification factor. Quantification factor includes errors and error changes, which are expressed as $K_{e}$ and $K_{\text {ec }}$, respectively. Formula(1) and Formula(2) can be used to confirm the value

$$
\begin{aligned}
K_{e} & =\frac{n}{x_{e}} \\
K_{e c} & =\frac{m}{X_{e c}}
\end{aligned}
$$

When actual value of errors is $\mathrm{e}_{1}, \alpha_{1}$ can be obtained through $\alpha_{1}=K_{e} e_{1}$. If $\alpha_{1}$ is integer, $\mathrm{e}_{1}$ is kept in the standard domain of discourse; If not, after it turns into integer, it is converted to the standard domain of discourse. For deviation change rate ec, the same method can be utilized to quantify it. Fuzzy reasoning based on the fuzzy logic converts a precondition into a conclusion. Numerous methods can realize the goal, and commonest one is the Mamdani reasoning method. The output result is the fuzzy vector. The accurate vector can be obtained after fuzzy judgment.

\section{Establishment of Load Forecasting Model of Power System Based on RBF Neural Network and Fuzzy Theory}

With historical load data and confirmed input samples, input data are preprocessed. Moreover, error data are settled and deficient data are supplemented in line with rules. Then with data normalization process and avoiding saturation of neurone, computing Formula (3) and Formula (4) are utilized to obtain normalization data. Parameters of RBF neural network are confirmed in line with self-organized learning selection center introduced in the learning algorithm. The confirmed center may be movable. Moreover, variance and learning weight can be confirmed in line with the center, so as to establish the RBF neural network model.

$$
\begin{gathered}
x_{m}=\frac{x_{\max }-x_{\min }}{2} \\
y=\frac{2\left(x-x_{m}\right)}{x_{\max }-x_{\min }}
\end{gathered}
$$

Load of power system is impacted by a great number of external factors and is equipped with uncertainty. Fuzzy control theory is utilized to improve the method of load forecasting, thus it can improve forecasting precision. For the established RBF neural network model, fuzzy control theory is introduced. According to the forecasting error and error change rate of neurone, the next adjusted weight is confirmed, so as to reduce forecasting errors. 
Input of fuzzy controller is the error e between predicted value and expected value, as well as error change rate ec. Moreover, ec $=\mathrm{e}(\mathrm{t})-\mathrm{e}(\mathrm{t}-1)$ and its correction is:

$$
\Delta U=\alpha e-(1-\alpha) e_{c}
$$

The fuzzy correction process is shown as the Figure 4.

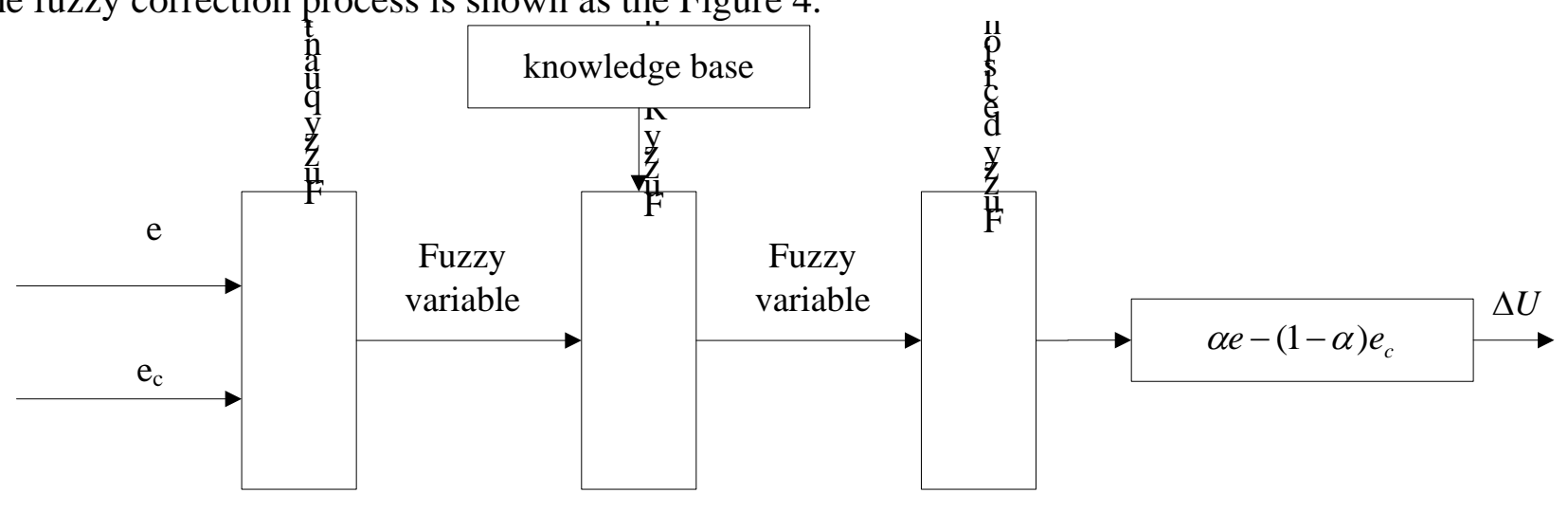

Fig. 4 Fuzzy Correction Diagram

The double-input and single-output fuzzy controller is selected. Error e and error change rate ec are regarded as the input. Moreover, fuzzy linguistic variable is expressed as $\bar{E}$ and $\bar{E}_{c}$. The linguistic variable is selected as positive big (PB), positive middle (PM), positive small (PS), zero (Z, negative small (NS), negative middle (NM), and negative big (NB). The fuzzy domain of discourse is $\bar{E}$ and $\bar{E}_{c}$, which are quantified as : $\mathrm{E}=\{-3,-2,-1,0,1,2,3\} ; \mathrm{E}_{\mathrm{c}}=\{-3,-2,-1,0,1,2,3\}$. The correction factor refers to the weight degree between error e and error change rate ec and is expressed as fuzzy linguistic variable $\alpha$. The fuzzy set is VS, S, M, B, and VB. The corresponding dot set is: 0 , $0.25,0.5,0.75,1.0$. The linguistic value of fuzzy linguistic value is the subset of the domain of discourse and finally is described by membership function. The model established in the paper is the membership function with the triangle function. Linguistic variables of error and error change rate are expressed as $\bar{E}$ and $\bar{E}_{c}$.

Fuzzy controlled rules are the summary on controlled experience and are the set of fuzzy conditional sentences. Fuzzy rules adjust correction factor. Rules are confirmed as follows: to adjust correction factor. The state table of fuzzy control controlled rules is shown in the Table 1. Table 1 State Table of Fuzzy Rules

\begin{tabular}{llllllll}
\hline & NB & NM & NS & Z & PS & PM & PB \\
\hline NB & VB & M & S & VS & S & M & VB \\
NM & VB & M & S & VS & S & M & VB \\
NS & VB & B & B & VS & B & B & VB \\
Z & VB & B & B & M & B & B & VB \\
PS & VB & B & B & VS & B & B & VB \\
PM & VB & M & S & VS & S & M & VB \\
PB & VB & M & S & VS & S & M & VB \\
\hline
\end{tabular}

The result obtained from fuzzy reasoning is the fuzzy quantity, which will be converted to precision quantity through fuzzy judgment. The Mamdani reasoning method and maximal membership method are utilized to obtain the LUT of fuzzy judgment. Before fuzzy judgment, it is necessary to quantify input. Then precision value can be obtained through LUT. Formula (5) is utilized to obtain adjustment of forecasting.

Based on the establishment of RBF neural network, fuzzy control is added to adjust and it can improve precision of load forecasting effectively. The specific forecasting procedures are shown in Figure 5. 


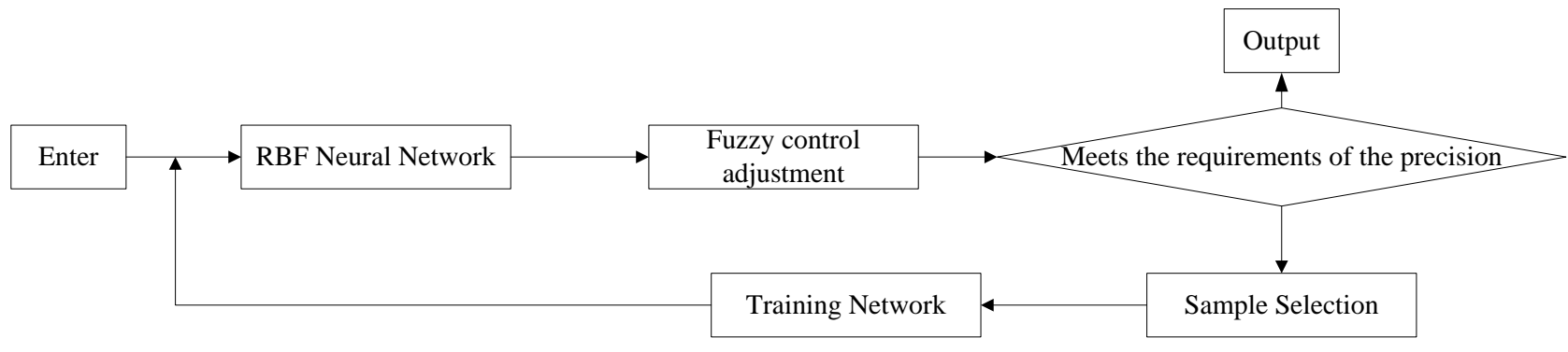

Fig. 5 Flow Chart Combining RBF Neural Network with Fuzzy Control Theory

\section{Example Algorithm Analysis}

After input data are preprocessing and normalization, RBF neural network model is established. Then, fuzzy control is added to adjust. Neural network of MATLAB software tool cabinet and fuzzy logic module are utilized to do power load forecasting in the area. The result is shown as Table 2 and Figure 6.

Table 2 Forecasting Results of Load Forecasting Based on RBF Neural Network and Fuzzy Control

\begin{tabular}{lrcl}
\hline Predicted nodes & $\begin{array}{r}\text { Actual } \\
\text { value(MW) }\end{array}$ & $\begin{array}{c}\text { Predicated } \\
\text { value(MW) }\end{array}$ & Relative error(\%) \\
\hline 1 & 72187 & 72142.96 & 0.061 \\
2 & 81443 & 80973.01 & 0.577 \\
3 & 78354 & 78915.95 & -0.717 \\
4 & 78226 & 78205.93 & 0.026 \\
5 & 73994 & 73875.77 & 0.16 \\
6 & 68744 & 68580.61 & 0.238 \\
7 & 70272 & 70124.91 & 0.209 \\
8 & 78321 & 78384.27 & -0.081 \\
9 & 76437 & 76360.56 & 0.1 \\
10 & 75714 & 76381.89 & -0.882 \\
11 & 73169 & 72815.3 & 0.483 \\
12 & 83775 & 83475.36 & 0.358 \\
13 & 72258 & 72302.6 & -0.062 \\
14 & 73193 & 73071.92 & 0.165 \\
15 & 73468 & 74481.69 & -1.38 \\
\hline
\end{tabular}

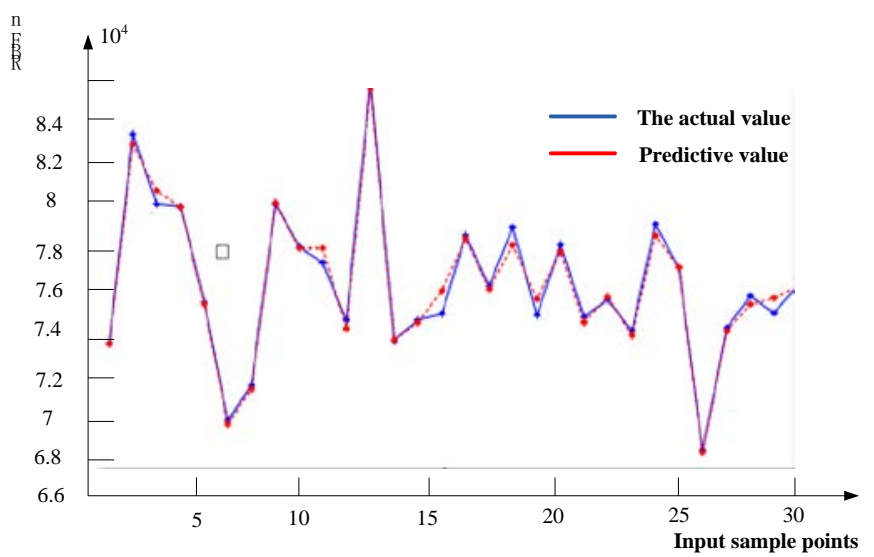

Fig. 6 Predicted Output and Actual Output Curves Combining RBF Neural Network and Fuzzy Control

It can be observed from Table 2 and Figure 6 that average relative error is $0.385 \%$. The result shows that predicted result error is smaller by comparing with the model established by using RBF neural network, proving that adjustment of fuzzy control theory is contributed to reducing predicted 
error and also indicating that the method combining fuzzy control theory with neural network has practical significance.

\section{Conclusions}

Load forecasting of power system is significance on dispatching power system, is the main basis of formulating electricity generation and power transmission plans, and also is an important aspect of modern development in power market. Improvement of precision in load forecasting of power system can improve economic benefits of electric power department effectively and promote safe and economic operation of grid. Load characteristics in different areas are different. Moreover, various methods of load forecasting have their respective advantages and disadvantages. Therefore, it is hard to find a suitable method that is appropriate for all load forecasting, but it is necessary to select a suitable forecasting method or integrate with several different methods to predict, so as to acquire higher forecasting precision. Based on the input samples of historical load data in an area, the biggest load forecasting is conducted in the area. The output result is conducted error analysis. Results obtained from these several methods are compared on the basis of load forecasting. The combined establishment of RBF neural network and fuzzy control has the minimum forecasting results. The forecasting effects are better, indicating that this method has realistic significance on load forecasting of actual power system.

\section{References}

[1] Niu Dongxiao, Wang Jianjun, Li Li and Li Cunbin, Short-term Load Forecasting Methods of Adaptive Neural Network Based on Rough Set and Decision-Making Tree, Electric Power Automation Equipment, Vol10, 2009, pp.30-34;

[2] Li Zuo, Zhou Buxiang and Li Nan, Daily Load Characteristic Curve Classification Based on Fuzzy Clustering and Improved BP Algorithm and Short-term Load Forecasting Power System Protection and Control, Vol.40(3), 2012, pp. 56-60;

[3] Zhao Dengfu, Wang Meng and Zhang Jiangshe, et al., Short-term Load Forecasting Based on Support Vector Machine, Proceedings of the CSEE, Vol. 22, 2002, pp. 26-30;

[4] Mao Li, Wang Yuntao and Liu Xingyang et al, Short-term Power Load Forecasting Methods Based on the Improved Limit Learning Machine[J], Power System Protection and Control, Vol. 40(20), 2012, pp. 140-144;

[5] Zhao Xizheng, Analysis and Forecasting of Chinese Power Load Characteristics, Beijing: China Electric Power Press, 2002, pp. 49-53;

[6] Zhou Hui, Gao Qi, He Jinghan, and Niu Wenjie, Teaching Study on Power Load Forecasting Knowledge System, Journal of Electric Power, 2006, pp. 25-26;

[7] Zhang Lin, Liu Xianshan and Yin Hejun, Application of Support Vector Machine in Load Forecasting Based on Time Sequence, Grid Technology, Vol., 28(19), 2004, pp.38-41;

[8] Li Yuancheng, Fang Tingjian and Zheng Guoxiang, The Study on Wavelet Support Vector Machine of Short-term Power Load Forecasting, Journal of University of Science and Technology of China, Vol.33(6), 2003, pp.726-732;

[9] Zhao Yuhong, Xiao Jinfeng and Chen Zhongze, Application of Mixed Fuzzy Neural Network in Short-term Load Forecasting, Journal of Power System and Automation, Vol.18(2), 2006, pp.99-104;

[10] Zhu Zhihui, Sun Yunlian and Ji Yu, Short-term Load Forecasting Based on Empirical Mode Decomposition and Least Squares Support Vector Machine, Relay, Vol. 35(8), 2007, pp. 37-40 